\title{
Субмонослойные квантовые точки InGaAs/GaAs, выращенные методом МОС-гидридной эпитаксии
}

\author{
(C) В.Я. Алешкин ${ }^{1}$, Н.В. Байдусь ${ }^{2}$, А.А. Дубинов ${ }^{1, \uparrow}$, К.Е. Кудрявцев ${ }^{1}$, С.М. Некоркин ${ }^{2}$, \\ А.В. Круглов ${ }^{2}$, Д.Г. Реунов ${ }^{2}$ \\ ${ }^{1}$ Институт фозики микроструктур Российской академии наук, \\ 603950 Нижний Новгород, Россия \\ ${ }^{2}$ Нижегородский государственный университет им. Н.И. Лобачевского, \\ 603950 Нижний Новгород, Россия \\ I E-mail: sanya@ipmras.ru
}

Поступила в Редакцию 1 апреля 2019 г.

В окончательной редакции 11 апреля 2019 г.

Принята к публикации 11 апреля 2019 г.

Проведен подбор режима роста квантовых точек методом МОС-гидридной эпитаксии для лазерных структур, выращенных на неотклоненных и отклоненных на $2^{\circ}$ подложках GaAs и излучающих на длинах волн выше 1.2 мкм при комнатной температуре. В результате экспериментов удалось достичь плотности квантовых точек $4 \cdot 10^{10} \mathrm{~cm}^{-2}$. В лазерных структурах с 7 слоями квантовых точек наблюдалось стимулированное излучение на длине волны 1.06 мкм при температуре жидкого азота. Пороговая плотность мощности оптической накачки составила около 5 кВт/см².

Ключевые слова: квантовые точки, МОС-гидридная эпитаксия, GaAs/InGaAs.

DOI: 10.21883/FTP.2019.08.48011.9124

\section{1. Введение}

В настоящее время в мире бурно развиваются исследования в области гибридных лазеров, т.е. когда лазерная структура выращена на кремниевой подложке. Такой лазер необходим для оптических межсоединений в области длин волн прозрачности объемного кремния (длина волны $>1.18$ мкм при комнатной температуре) [1]. В лазерах с квантовыми ямами (КЯ) InGaAs/GaAs для смещения рабочей длины волны в область прозрачности объемного кремния требуется увеличение доли In в КЯ либо увеличение толщины КЯ. Однако в обоих случаях рост упругих напряжений сжатия в КЯ приводит к образованию на гетероинтерфейсе InGaAs/GaAs дислокаций несоответствия. Известным приемом, позволяющим увеличить энергию образования дислокаций несоответствия и подавить их формирование, является встраивание вблизи КЯ тонких слоев GaAsP [2]. Для лазерных структур, выращенных на кремнии, удалось лишь несколько снизить степень релаксации упругих напряжений в КЯ, однако подавить релаксацию целиком не удалось, как это показано для структур на GaAsподложках [3]. В активной области лазера появляются в высокой концентрации центры безызлучательной рекомбинации, что сопровождается резкой деградацией люминесцентных свойств получаемых структур. Кроме того, структуры с КЯ InGaAs на кремниевых подложках очень чувствительны к дефектам, которые возникают на границе кремния и полупроводников $\mathrm{A}^{\mathrm{III}} \mathrm{B}^{\mathrm{V}}$ [4-6]. Поэтому в последнее время для преодоления этих двух проблем в качестве активной среды используются квантовые точки (КT) InAs.
К настоящему времени был создан ряд лазерных диодов с КТ на основе полупроводников $\mathrm{A}^{\mathrm{III}} \mathrm{B}^{\mathrm{V}}$, выращенных на $\mathrm{Si}$ подложках, отклоненных на несколько градусов от направления [001], для подавления формирования антифазных дефектов, вызванных ростом полярных материалов на неполярной подложке $[7,8]$. В то же время существующие технологии изготовления кремниевых процессоров развиты для точно ориентированных подложек $\mathrm{Si}$ (001) с отклонением, не превышающим $0.5^{\circ}$ [9]. В последние годы появились сообщения о формировании лазеров с KT InAs на неотклоненных кремниевых подложках $[10,11]$ методом молекулярнопучковой эпитаксии (МПЭ), использование которого не является практичным с точки зрения промышленного производства, и лишь совсем недавно появилась первая работа, посвященная формированию лазера с КТ на кремниевой подложке более высокопроизводительным, промышленным методом МОС-гидридной эпитаксии [12]. Однако авторы этой работы использовали отклоненную на $4^{\circ}$ подложку. Поэтому до сих пор является актуальным рост гибридной лазерной структуры с КТ на неотклоненной кремниевой подложке методом MOC-гидридной эпитаксии. Работ, посвященных лазерам на КТ, выращенных методом МОС-гидридной эпитаксии на подложках $\mathrm{GaAs}$ и излучающим на длинах волн выше 1.2 мкм, также мало [13,14].

В настоящей работе сообщается о проведении ростовых экспериментов, направленных на подбор оптимальных режимов роста КТ методом МОС-гидридной эпитаксии на неотклоненных и отклоненных на $2^{\circ}$ подложках GaAs для реализации лазерных структур, излучающих на длинах волн выше 1.2 мкм. 
Режимы роста исследуемых структур InGaAs/GaAs

\begin{tabular}{c|c|c|c|r|c|c}
\hline $\begin{array}{c}\text { Режим } \\
\text { роста }\end{array}$ & $d_{\mathrm{In}}, \mathrm{MC}$ & $d_{\mathrm{Ga}}, \mathrm{MC}$ & $F\left(\mathrm{AsH}_{3}\right)$ & Ncycle & $D_{\Sigma}, \mathrm{MC} / \mathrm{HM}$ & $T_{\mathrm{gr}},{ }^{\circ} \mathrm{C}$ \\
\hline $\mathrm{A}$ & 0.5 & 2 & + & 5 & $13 / 3.9$ & 520 \\
$\mathrm{~B}$ & 1 & 1 & + & 5 & $10 / 3$ & 520 \\
$\mathrm{C}$ & 0.25 & 0.25 & - & 10 & $5 / 1.5$ & 515 \\
$\mathrm{D}$ & 0.25 & 0.25 & - & 10 & $5 / 1.5$ & 485
\end{tabular}

Указаны: количество индия и галлия $\left(d_{\mathrm{In}}\right.$ и $\left.d_{\mathrm{Ga}}\right)$, осаждаемого в каждом цикле субмонослойного роста и число таких циклов $\left(N_{\text {cycle }}\right)$ при росте одного слоя КТ; суммарная толщина осаждаемого материала $\left(D_{\Sigma}\right)$, температура роста $\left(T_{\mathrm{gr}}\right)$. Поток арсина $(F)$ сохранялся неизменным в течение всего ростового цикла $\left(,+^{*}\right)$, либо выключался во время осаждения In и $\mathrm{Ga}(,--“)$

\section{2. Исследуемые образцы и методика субмонослойного роста КТ}

Исследуемые гетероструктуры выращивались методом MOC-гидридной эпитаксии при пониженном давлении на установке Aixtron AIX-200RF. Рост проводился на подложках GaAs как с точной ориентацией (001), так и с отклонением $2^{\circ}$ в направлении [011]. Источниками элементов являлись триметилгаллий, триметилиндий, триметилалюминий и арсин. Рост буферного слоя GaAs, ограничительного слоя $\mathrm{AlGaAs}$ и вновь слоя $\mathrm{GaAs}$ (каждый из этих слоев имел толщину 0.5 мкм) осуществлялся при температуре $T_{\mathrm{gr}} \sim 650^{\circ} \mathrm{C}$. Рост непосредственно КТ проводился при пониженной температуре $\left(485-520^{\circ} \mathrm{C}\right)$ в циклическом режиме субмонослойного осаждения [15]. В каждом цикле последовательно осаждались индий (в количестве 0.25-1 монослоя, МС) и галлий (0.25-2 МС), после чего делалась пауза в 5 с; далее такой цикл повторялся 5-10 раз. Поток арсина был при этом неизменным либо прерывался на время осаждения In и $\mathrm{Ga}$. Всего было опробовано 4 различных ростовых режима, их параметры приведены в таблице. После роста слоя КТ структура заращивалась покровным слоем GaAs толщиной 20-200нм при той же (низкой) температуре, при которой проводился рост КТ.

Выращивались также многослойные структуры, содержащие 3 слоя КТ, разделенных спейсерными слоями $\mathrm{GaAs}$ толщиной 6-12 нм; при этом последний слой поверхностных КТ заращивался тонким (2 мкм) покровным слоем GaAs. Такие комбинированные структуры позволили проводить характеризацию формируемых массивов КТ как с применением атомно-силовой микроскопии (ACM), так и с использованием спектроскопии фотолюминесценции (ФЛ).

Исследование морфологии поверхности выращенных образцов и установление характеристик получаемых массивов КТ InGaAs/GaAs проводились с помощью ACM-микроскопа Solver PRO-M („NT-MDT“, Россия) на воздухе в полуконтактном режиме; при этом использовались зонды NT-MDT ETALON HA_HR с радиусом закругления не более 10 нм. По АCМ-изображениям определялись поверхностная концентрация, высота и латеральные размеры КТ. Спектры ФЛ выращенных структур измерялись с помощью монохроматора МДР23 , оснащенного германиевым фотодетектором, с применением методики синхронного детектирования сигнала. Возбуждение ФЛ осуществлялось непрерывным лазером с длиной волны излучения 0.63 мкм, либо, при импульсных измерениях, с помощью параметрического генератора света „Spectra-Physics“ MOPO-SL (длина волны излучения 0.8 мкм, длительностью импульса 10 нс, частота повторения импульсов 10 Гц). Измерения проводились при температурах $T=77$ и $300 \mathrm{~K}$.

\section{3. Экспериментальные результаты и обсуждение}

Спектры ФЛ образцов \# 1418, \#1419 и \#1420, выращенных в режимах $\mathrm{A}, \mathrm{B}$ и $\mathrm{C}$ соответственно (см. таблицу) на подложках (001)GaAs, приведены на рис. 1. В спектре ФЛ образца \# 1418 наблюдается односительно узкий пик $(\delta \lambda \sim 35$ нм при $T=300 \mathrm{~K})$ вблизи длины волны 1 мкм, что указывает на реализацию в режиме А двумерного роста и формирование квантовой ямы InGaAs. Отметим, что в работе [16] при росте методом МПЭ сходные условия субмонослойного осаждения материалов приводили к формированию КT InAs/GaAs. Увеличение доли In, осаждаемого в одном цикле (образец \# 1419, выращенный в режиме В), приводит к возникновению в спектре ФЛ, наряду с коротковолновым узким пиком, также и широкого пика $(\delta \lambda \sim 100$ нм при $T=300 \mathrm{~K})$ с максимумом вблизи 1.2 мкм. Можно сделать вывод, что в этом случае происходит переход от двумерного роста к трехмерному, а наблюдаемые

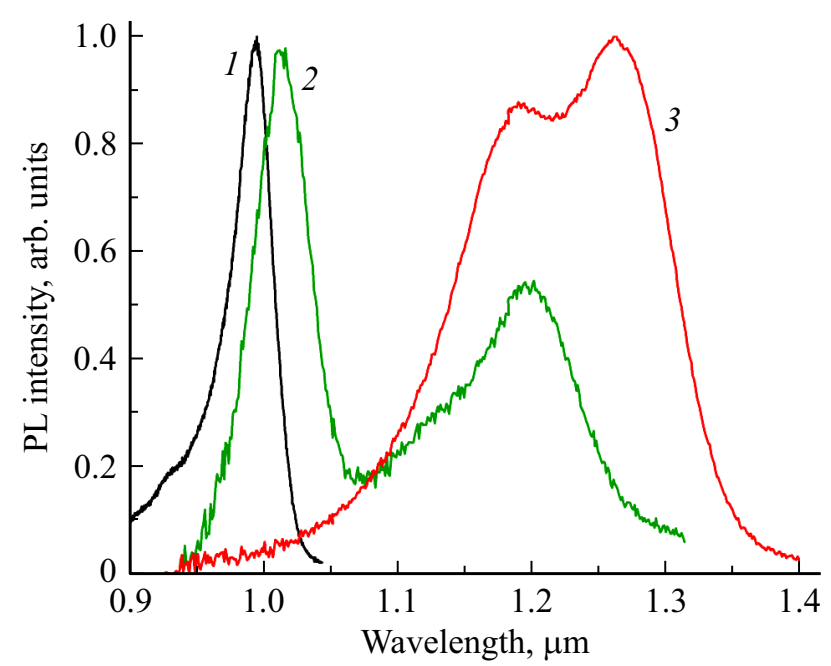

Рис. 1. Спектры ФЛ образцов, выращенных в различных режимах субмонослойного осаждения InGaAs: 1 - режим A (образец \# 1418), 2 - режим В (\#1419), 3 - режим С (\# 1420). Вид спектров ФЛ в серии \# 1418/\# 1419/\# 1420 отражает переход от двумерного роста (КЯ) через „промежуточное состояние“ (КЯ + КТ) к трехмерному (КТ). Температура измерений $T=300 \mathrm{~K}$. 

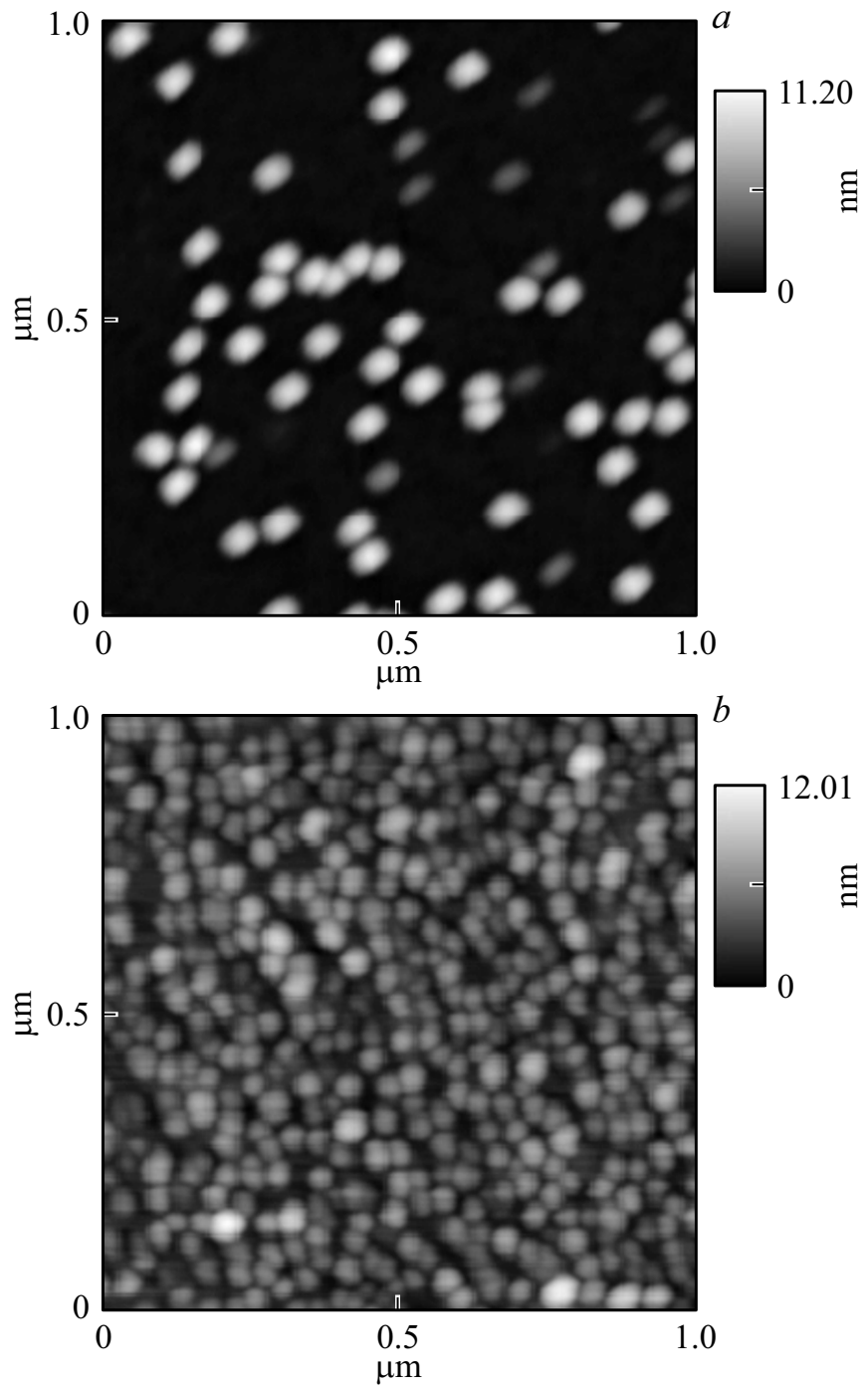

Рис. 2. АСМ-изображение поверхностных КТ образцов \# 1420 (a) и \# 1462-0 (b). Размер снимков $1 \times 1$ мкм.

в спектрах ФЛ полосы эмиссии соответствуют излучению квантовой ямы („смачивающего“ слоя) и образующихся КТ.

Последующая оптимизация режимов роста заключалась в уменьшении количества осаждаемого в каждом цикле материала (режимы C и D); при этом во время осаждения металлических элементов выключался поток арсина. В этом случае в спектрах ФЛ наблюдалась одна широкая полоса $(\delta \lambda>100$ нм) эмиссии ФЛ с максимумом на длине волны 1.26 мкм, обусловленная межзонными переходами в КТ (рис. 1, образец \# 1420). Коротковолновое плечо в спектре ФЛ с максимумом вблизи 1.19 мкм обусловлено оптическими переходами с участием возбужденных состояний, что подтверждается зависимостью вида спектра от плотности мощности накачки (не приводится в данной работе) [17]. Формирование КТ подтверждается и данными АСМ-исследований; снимок поверхности образца \#1420 представлен на рис. 2, $a$.
Наблюдается достаточно однородный массив КТ с поверхностной плотностью $\sim 8 \cdot 10^{9} \mathrm{~cm}^{-2}$. Кроме КТ, на поверхности образца присутствуют также большие (повидимому, с образованием дислоцикаций) островки с поверхностной плотностью $\sim 10^{8} \mathrm{~cm}^{-2}$. Уменьшение температуры роста КТ до $T_{\mathrm{gr}} \sim 485^{\circ} \mathrm{C}$ (режим $\mathrm{D}$, образец \# 1462-0) позволило одновременно снизить плотность крупных кластеров до $10^{7} \mathrm{~cm}^{-2}$ и существенно, до $4 \cdot 10^{10} \mathrm{~cm}^{-2}$, повысить поверхностную плотность КТ. Соответствующий АСМ-снимок представлен на рис. $2, b$; видно, что как латеральный размер, так и высота КТ значительно уменьшились в сравнении со случаем высокотемпературного роста.

Ростовые эксперименты проводились также и на вицинальных (отклоненных на $2^{\circ}$ в направлении [011]) подложках GaAs (образец \# 1462-2); при этом также было достигнуто формирование КТ приблизительно с той же концентрацией, что и в случае роста на сингулярной поверхности (001)GaAs. Латеральные размеры и высота КТ при росте на вицинальной поверхности оказалась несколько меньше, чем для роста на (001)GaAs. Спектры ФЛ образцов \# 1462-0 и \# 1462-2 сопоставлены на рис. 3 , и следует отметить два их существенных различия. Вопервых, максимум излучения КТ в образце \#1462-2 смещен на $\sim 100$ нм в область меньших длин волн, что коррелирует с уменьшением размера КТ; во-вторых, в спектре ФЛ этого образца при температуре $T=77 \mathrm{~K}$ проявляется коротковолновый пик вблизи 1 мкм, который может быть связан с наличием неоднородного квазидвумерного смачивающего слоя либо массива мелких КТ. Указанные обстоятельства позволяют сделать заключение, что наличие ступеней роста на вицинальной поверхности задерживает переход от двумерного роста слоя InGaAs к трехмерному и в некоторой степени препятствуют формированию КТ. В то же время рассмотрение роста структур с КТ на отклоненных под-

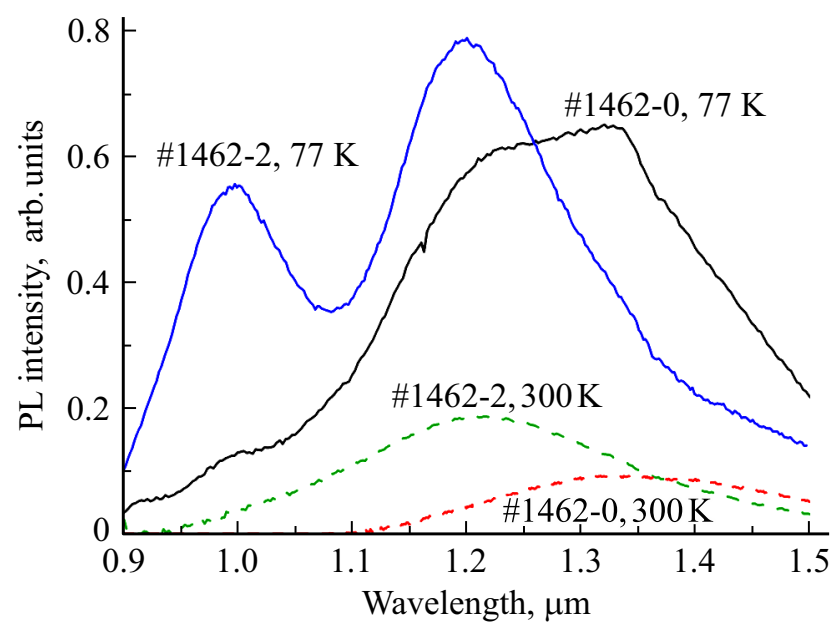

Рис. 3. Спектры ФЛ образцов \# 1462-0 и \# 1462-2, измеренные при температуре $T=77 \mathrm{~K}$ (сплошные кривые) и $T=300 \mathrm{~K}$ (штриховые кривые). 
ложках может быть важно для последующей реализации гибридных лазерных структур с КТ $\mathrm{A}^{\mathrm{III}} \mathrm{B}^{\mathrm{V}}$ на кремнии.

В данной работе были реализованы лазерные гетероструктуры с KT InGaAs/GaAs на подложках GaAs. Как и в случае структур, предназначенных для отработки условий роста и наблюдения спонтанной ФЛ, рост лазерных структур осуществлялся на точно ориентированных (001) подложках и на подложках, отклоненных на $2^{\circ}$ в направлении [011]. В лазерных структурах формировался толстый (1 мкм) нижний слой оптического ограничения $\mathrm{Al}_{0.8} \mathrm{Ga}_{0.2} \mathrm{As}$, далее осаждался волноводный слой GaAs толщиной 200 нм, в центр которого встраивались 7 слоев КТ с расстоянием между слоями 12 нм. Структура закрывалась тонким (50 нм) слоем $\mathrm{Al}_{0.3} \mathrm{Ga}_{0.7} \mathrm{As}$ c целью снижения поверхностной рекомбинации фотовозбужденных носителей заряда и покровным слоем GaAs, толщиной также 50 нм. Отметим, что для обеспечения хорошего структурного и оптического качества верхнего ограничивающего слоя $\mathrm{AlGaAs}$ необходим рост при достаточно высокой температуре $\left(T_{\mathrm{gr}} \sim 600-650^{\circ} \mathrm{C}\right)$, превышающей температуру роста КТ, что оказывает значительное влияние на излучательные свойства получаемых структур. Так, на рис. 4 сопоставлены спектры ФЛ образцов \# 1452-0 и \# 1452-2 (лазерные структуры, выращенные на сингулярной и вицинальной подложках соответственно) со спектрами ФЛ образцов \# 1462-0 и \# 1462-2. Рост этих образцов проводился в одинаковых условиях, за исключением роста верхнего ограничивающего слоя. Сопоставление приведенных спектров ФЛ позволяет сделать вывод, что высокотемпературное воздействие при заращивании слоев КТ приводит к существенному сдвигу длины волны излучения получаемых массивов КТ в коротковолновую часть спектра, что может объясняться взаимодиффузией материалов КТ InGaAs и матрицы GaAs [18]. Данное обстоятельство

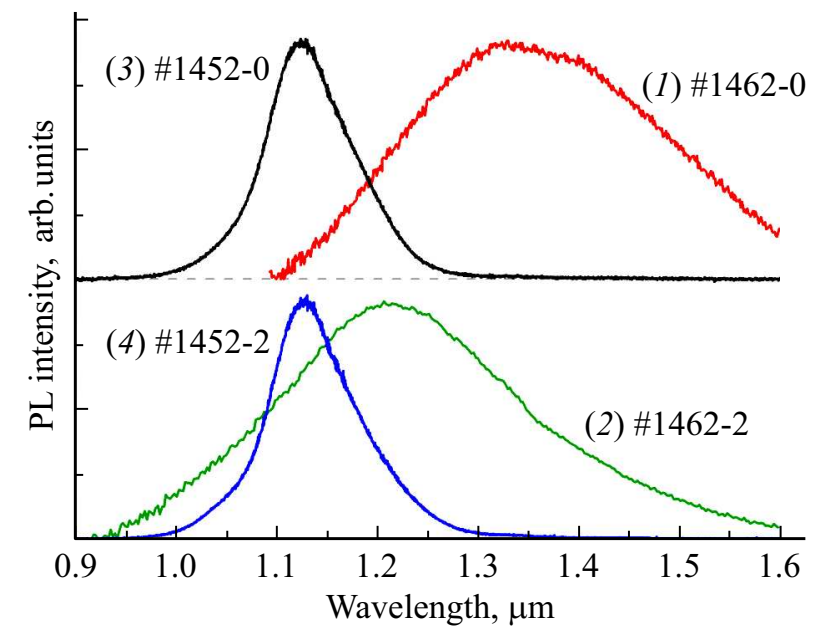

Рис. 4. Спектры ФЛ образцов с КТ InGaAs/GaAs \# 1462-0 (1) и \#1462-2 (2), а также лазерных структур \#1452-0 (3) и \# 1452-2 (4). Спектры нормированы на максимальное значение и разнесены по вертикали для наглядности. Измерения при температуре $T=300 \mathrm{~K}$.

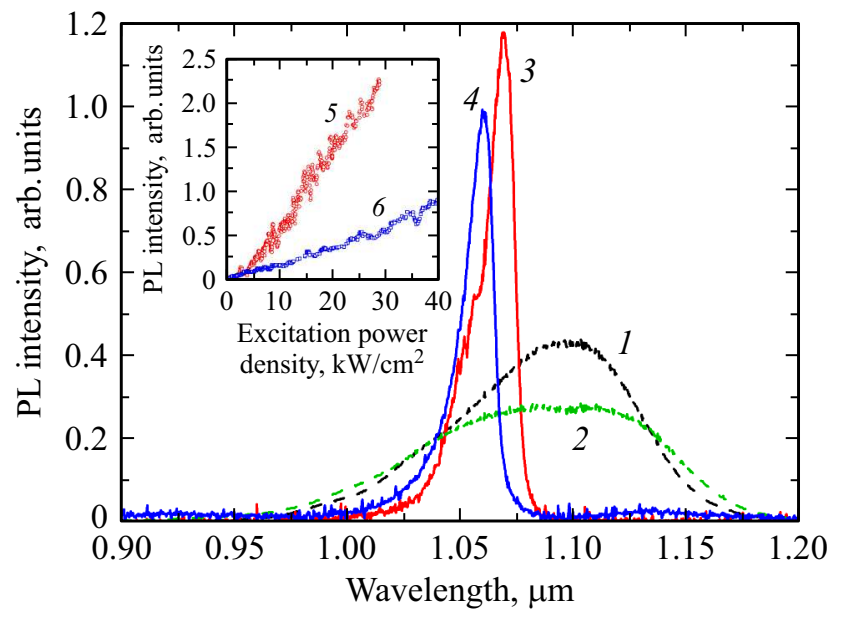

Рис. 5. Спектры излучения образцов \#1452-0 $(1,3)$ и \# 1452-2 $(2,4)$ при непрерывной $(1,2)$ накачке (штриховые кривые) и при импульсной $(3,4)$ накачке (сплошные кривые). На вставке - зависимость максимума интенсивности излучения от плотности мощности накачки для образцов \# 1452-0 (5) и \# 1452-2 (6). Температура измерений $T=77 \mathrm{~K}$.

определяет интерес к развитию низкотемпературного роста ограничительных слоев в лазерных структурах с KT InGaAs/GaAs; в частности, такие слои, осаждаемые при температуре роста КТ без потери качества, могли бы быть реализованы на основе InGaP [12].

В лазерных структурах \# 1452-0 и \# 1452-2 в условиях импульсной оптической накачки удалось наблюдать появление стимулированного излучения на межзонных переходах в КТ. Соответствующие спектры спонтанной эмиссии и стимулированного излучения, измеренные при температуре $T=77 \mathrm{~K}$, приведены на рис. 5. Для обоих образцов пик стимулированного излучения сдвинут в сторону высоких энергий относительно пика спонтанного излучения и хорошо соответствует по спектральному положению области излучательных переходов с участием возбужденных состояний КТ, что свидетельствует об их участии в обеспечении оптического усиления. На вставке рис. 5 приведены зависимости максимума интенсивности эмиссии от плотности мощности накачки для образцов \# 1452-0 и \# 1452-2. Пороговые плотности мощности накачки составили порядка $5 \kappa \mathrm{BT} / \mathrm{cm}^{2}$ и $10 \mathrm{\kappa} \mathrm{BT} / \mathrm{cm}^{2}$ соответственно для образцов, выращенных на точно ориентированной и отклоненной подложках GaAs.

\section{4. Заключение}

Были выявлены режимы субмонослойного роста, обеспечивающие формирование КT InGaAs/GaAs, излучающих на длинах волн выше 1.2 мкм при комнатной температуре, в процессе МОС-гидридной эпитаксии на неотклоненных $(001)$ и отклоненных на $2^{\circ}$ подложках GaAs. Плотность КT составила $\sim 4 \cdot 10^{10} \mathrm{~cm}^{-2}$ при тем- 
пературе роста $485^{\circ} \mathrm{C}$; при этом плотность дислоцированных кластеров составила меньше $10^{7} \mathrm{~cm}^{-2}$. Выращены лазерные структуры с 7 слоями КТ InGaAs/GaAs, в которых наблюдалось стимулированное излучение на длине волны 1.06 мкм при температуре жидкого азота.

\section{Благодарности}

В работе использовалось оборудование УСУ „Фемтоспектр“ ЦКП „Физика и технология наноструктур“ Института физики микроструктур Российской академии наук.

\section{Финансирование работы}

Работа частично выполнена при поддержке гранта Российского фонда фундаментальных исследований № 18-29-20016 и в рамках государственного задания Института физики микроструктур РАН на 2019 г. (№ 00352019-0020-C-01).

\section{Конфликт интересов}

Авторы заявляют, что у них нет конфликта интересов.

\section{Список литературы}

[1] C. Sun, M.T. Wade, Y. Lee, J.S. Orcutt, L. Alloatti. Nature, 528, 534 (2015).

[2] Д.А. Винокуров, Д.Н. Николаев, Н.А. Пихтин, А.Л. Станкевич, В.В Шамахов, А.Д. Бондарев, Н.А. Рудова, И.С. Тарасов. ФТП, 45 (9), 1274 (2011).

[3] Н.В. Байдусь, В.Я. Алешкин, А.А. Дубинов, З.Ф. Красильник, К.Е. Кудрявцев, С.М. Некоркин, А.В. Новиков, А.В. Рыков, Д.Г. Реунов, М.В. Шалеев, П.А. Юнин, Д.В. Юрасов. ФТП, 52 (12), 1443 (2018).

[4] V.Ya. Aleshkin, N.V. Baidus, A.A. Dubinov, A.G. Fefelov, Z.F. Krasilnik, K.E. Kudryavtsev, S.M. Nekorkin, A.V. Novikov, D.A. Pavlov, I.V. Samartsev, E.V. Skorokhodov, M.V. Shaleev, A.A. Sushkov, A.N. Yablonskiy, P.A. Yunin, D.V. Yurasov. Appl. Phys. Lett., 109, 061111 (2016).

[5] N.V. Kryzhanovskaya, E.I. Moiseev, Yu.S. Polubavkina, M.V. Maximov, M.M. Kulagina, S.I. Troshkov, Yu.M. Zadiranov, A.A. Lipovskii, N.V. Baidus, A.A. Dubinov, Z.F. Krasilnik, A.V. Novikov, D.A. Pavlov, A.V. Rykov, A.A. Sushkov, D.V. Yurasov, A.E. Zhukov. Opt. Express, 25, 16754 (2017).

[6] N. Baidus, V. Aleshkin, A. Dubinov, K. Kudryavtsev, S. Nekorkin, A. Novikov, D. Pavlov, A. Rykov, A. Sushkov, M. Shaleev, P. Yunin, D. Yurasov, Z. Krasilnik. Crystals, 8, 311 (2018).

[7] A.Y. Liu, C. Zhang, J. Norman, A. Snyder, D. Lubyshev, J.M. Fastenau, A.W.K. Liu, A.C. Gossard, J.E. Bowers. Appl. Phys. Lett., 104, 041104 (2014).

[8] S. Chen, W. Li, J. Wu, Q. Jiang, M. Tang, S. Shutts, S.N. Elliott, A. Sobiesierski, A.J. Seeds, I. Ross, P.M. Smowton, H. Liu. Nature Photonics, 10, 307 (2016).

[9] K. Volz, A. Beyer, W. Witte, J. Ohlmann, I. N'emeth, B. Kunert, W. Stolz. J. Cryst. Growth, 315, 37 (2011).
[10] Y. Wan, J. Norman, Q. Li, M.J. Kennedy, D. Liang, C. Zhang, D. Huang, Z. Zhang, A.Y. Liu, A. Torres, D. Jung, A.C. Gossard, E.L. Hu, K.M. Lau, J.E. Bowers. Optics. 4, 940 (2017).

[11] A.Y. Liu, J. Peters, X. Huang, D. Jung, J. Norman, M.L. Lee, A.C. Gossard, J.E. Bowers. Optics. Lett., 42, 338 (2017).

[12] J. Wang, H. Hu, H. Yin, Y. Bai, J. Li, X. Wei, Y. Liu, Y. Huang, X. Ren, H. Liu. Photonics Res., 6, 321 (2018).

[13] S.M. Kim, Y. Wang, M. Keever, J.S. Harris. IEEE Photonics Technology Letters, 16, 377 (2004).

[14] I.N. Kaiander, R.L. Sellin, T. Kettler, N.N. Ledentsov, D. Bimberg, N.D. Zakharov, P. Werner. Appl. Phys. Lett., 84, 2992 (2004).

[15] S.S. Mikhrin, A.E. Zhukov, A.R. Kovsh, N.A. Maleev, V.M. Ustinov, Yu.M. Shernyakov, I.P. Soshnikov, D.A. Livshits, I.S. Tarasov, D.A. Bedarev, B.V. Volovik, M.V. Maximov, A.F. Tsatsul'nikov, N.N. Ledentsov, P.S. Kop'ev, D. Bimberg, Zh.I. Alferov. Semicond. Sci. Technol., 15, 1061 (2000).

[16] D. Arsenijevic, C. Liu, A. Payusov, M. Stubenrauch, D. Bimberg. IEEE Photon. Tech. Lett., 24 (11), 1041 (2012).

[17] D.L. Huffaker, D.G. Deppe. Appl. Phys. Lett., 73, 520 (1998).

[18] D. Franke, M. Moehrle, J. Boettcher, P. Harde, A. Sigmund, H. Kuenze. Appl. Phys. Lett., 91, 081117 (2007).

Редактор А.Н. Смирнов

\section{Submonolayer InGaAs/GaAs quantum dots grown by MOCVD}

V.Ya. Aleshkin ${ }^{1}$, N.V. Baidus ${ }^{2}$, A.A. Dubinov ${ }^{1}$, K.E. Kudryavtsev ${ }^{1}$, S.M. Nekorkin ${ }^{2}$, A.V. Kruglov ${ }^{2}$, D.G. Reunov ${ }^{2}$

${ }^{1}$ Institute for Physics of Microstructures, Russian Academy of Sciences, 603950 Nizhny Novgorod, Russia ${ }^{2}$ Lobachevsky State University of Nizhny Novgorod, 603950 Nizhny Novgorod, Russia

Abstract In this paper, we selected the growth mode of InGaAs quantum dots by MOCVD for laser structures grown on exact and deflected at $2^{\circ} \mathrm{GaAs}$ substrates and emit at wavelengths above $1.2 \mu \mathrm{m}$ at room temperature. As a result, the density of quantum dots $4 \cdot 10^{10} \mathrm{~cm}^{-2}$ was achieved. Stimulated emission was observed in laser structures with 7 layers of quantum dots at a wavelength of $1.06 \mu \mathrm{m}$ at liquid nitrogen temperature. The threshold power density of optical pumping was about $5 \mathrm{~kW} / \mathrm{cm}^{2}$. 